\title{
Expression of GaINAc-T3 and its relationships with clinicopathological factors in 61 extrahepatic bile duct carcinomas analyzed using stepwise sections-Special reference to its association with lymph node metastases-
}

\author{
Takahiro Inoue $^{1}$, Takashi Eguchi ${ }^{1}$, Yoshinao Oda ${ }^{1}$, Kenichi Nishiyama ${ }^{1,3}$, Kei Fujii ${ }^{1}$, \\ Hiroto Izumi ${ }^{4}$, Kimitoshi Kohno ${ }^{4}$, Koji Yamaguchi ${ }^{2}$, Masao Tanaka ${ }^{2}$ and \\ Masazumi Tsuneyoshi ${ }^{1}$
}

${ }^{1}$ Department of Anatomic Pathology, Graduate School of Medical Sciences, Kyushu University, Fukuoka, Japan; ${ }^{2}$ Department of Surgery and Oncology, Graduate School of Medical Sciences, Kyushu University, Fukuoka, Japan; ${ }^{3}$ Department of Pathology, National Kyushu Cancer Center, Fukuoka, Japan and ${ }^{4}$ Department of Molecular Biology, School of Medicine, University of Occupational and Environmental Health, Kitakyushu, Japan

Extrahepatic bile duct carcinomas (EBDCs) still result in an unfavorable prognostic outcome, and little is known about their biological aggressiveness. Recently, UDP- $N$-acetyl-alpha-D-galactosamine:polypeptide $N$-acetylgalactosaminyl transferase-3 (GalNAC-T3) was reported to be associated with differentiation and malignant potential of human carcinomas. Here, we investigated 61 EBDCs for their detailed clinicopathological features and GalNAc-T3 expression by immunohistochemistry, and then evaluated the relationships between the clinicopathological features and GaINAC-T3 expression patterns. Most of the EBDCs were massively invasive tumors with frequent vascular or perineural invasion and lymph node metastases. GaINAc-T3 expression was detected in all 61 EBDCs, and the expression patterns could be classified into granular and diffuse types. All four noninvasive or minimally invasive EBDCs were the granular type. Among the $\mathbf{5 8}$ minimally or massively invasive EBDCs, the GaINAC-T3 expression pattern at the luminal surface was the granular type in 38 cases $(66 \%)$ and diffuse type in 20 cases (34\%), while the expression pattern at the invasive front was the granular type in 26 cases (45\%) and diffuse type in 32 cases (55\%). Among the 38 cases with granular-type expression at the luminal surface, 26 cases $(68 \%)$ remained the granular type and 12 cases $(32 \%)$ became the diffuse type at the invasive front. All 20 cases with diffuse-type expression at the luminal surface remained the diffuse type at the invasive front. Diffuse-type GalNAc-T3 expression at the invasive front was significantly associated with lymph node metastasis $(P<0.05)$. There were no significant correlations between the GaINAc-T3 expression patterns and other clinicopathological factors, including tumor differentiation, depth of invasion or overall survival. In conclusion, EBDCs alter their GaINAc-T3 expression pattern during tumor growth, and the difference in the GaINAc-T3 expression pattern may be associated with lymph node metastasis. Clinically, preoperative evaluation of GaINAC-T3 expression is considered to be useful for decisions regarding operative procedures. Modern Pathology (2007) 20, 267-276. doi:10.1038/modpathol.3800700

Keywords: extrahepatic bile duct carcinoma; GalNAc-T3; O-linked glycoprotein; mucin; lymph node metastasis

Extrahepatic bile duct carcinoma (EBDC) is a relatively uncommon tumor worldwide, but is more common in Japan than in Western countries. ${ }^{1}$ Despite recent progress in diagnostic and therapeu-

Correspondence: Dr T Inoue, MD, Department of Anatomic Pathology, Graduate School of Medical Sciences, Kyushu University, 3-1-1 Maidashi, Higashi-ku, Fukuoka 812-8582, Japan.

E-mail: takinoue@surgpath.med.kyushu-u.ac.jp

Received 30 June 2006; accepted 17 August 2006 tic procedures, its prognostic outcome remains unfavorable. ${ }^{2,3}$ A number of EBDCs arise in bile ducts with an abnormal pancreatico-biliary duct junction, and their tumorigenesis is considered to be associated with inflow of pancreatic juice into the bile duct. ${ }^{4-7}$ However, more common mechanisms for the tumorigenesis and the reasons for the biological aggressiveness of EBDCs have not yet been sufficiently elucidated, and further investigations are required. 
In recent years, $O$-linked glycoproteins, such as mucin-type glycoproteins, have been reported to be associated with the differentiation and malignant potential, such as altered cell adhesion, invasion, metastasis, recurrence and prognosis, of adenocarcinomas. ${ }^{8} \mathrm{O}$-glycosylation of these glycoproteins has been considered to play a key role in determining the tumor characteristics. The initial glycosylation of mucin-type $O$-linked glycoproteins is catalyzed by a family of uridine diphosphate (UDP) $\mathrm{N}$-acetylalpha-D-galactosamine:polypeptide $N$-acetylgalactosaminyl transferases (GalNAc-Ts). ${ }^{9-15}$ A total of 15 human GalNAc-Ts have been identified to date, ${ }^{16}$ and observed differences in the $O$-linked glycoprotein expression patterns among tumors, which may affect tumor differentiation and progression, can be explained by differing expression levels or activities of these GalNAc-T subtypes. ${ }^{8,11}$ Among the GalNAc$\mathrm{T}$ subtypes, the expression of GalNAc-T3 is highly specific for glandular epithelia, and it is also expressed at high levels in human adenocarcinomas. ${ }^{17,18}$ Furthermore, recent studies have suggested a relationship between GalNAc-T3 expression and the tumor differentiation or progression, including prognosis, of human adenocarcinomas in the colon, lung, stomach, pancreas and gallbladder. ${ }^{24-30}$ Although the GalNAc-T subtypes are usually localized in the Golgi apparatus, their subcellular localization alters following malignant transformation due to reorganization of the Golgi apparatus elements. ${ }^{8,19,20}$ Therefore, not only the levels but also the patterns of GalNAc-T3 expression, such as the subcellular distribution, may be associated with the malignant transformation and differentiation of adenocarcinoma cells.

In the present study, the clinicopathological findings of 61 surgically resected EBDCs were evaluated in detail using whole stepwise sections, and their GalNAc-T3 expression levels and patterns were studied by immunohistochemistry using formalin-fixed paraffin-embedded sections. The correlations between the immunohistochemical expression patterns of GalNAc-T3 and the clinicopathological factors, mode of progression and prognosis were investigated to clarify the aggressiveness of EBDCs in association with GalNAc-T3 expression.

\section{Materials and methods}

\section{Tissue Specimens}

Surgical specimens were obtained from 61 patients who had undergone surgery for primary EBDCs. For pathological examination, the surgically resected specimens were fixed in $10 \%$ formalin at room temperature. After recording of the gross features of the tumors, such as the tumor size or growth pattern, each whole specimen was cut into slices at intervals of $0.5 \mathrm{~cm}$, and all the slices were routinely processed, embedded in paraffin, sectioned and micro- scopically evaluated for the histopathological characteristics. All the specimens in this study were diagnosed and filed as primary EBDCs at the Department of Anatomic Pathology, Graduate School of Medicine, Kyushu University during the period from June 1989 to September 2001.

The clinicopathological findings were evaluated according to the General Rules for Surgical and Pathological Studies on Cancer of the Biliary Tract ${ }^{21}$ with reference to the WHO classification, ${ }^{22,23}$ and staged according to the TNM classification of the UICC. ${ }^{23}$

Table 1 Clinicopathological factors of the EBDCs $(n=61)$

\begin{tabular}{|c|c|}
\hline \multicolumn{2}{|l|}{ Sex } \\
\hline Male & $46(75 \%)$ \\
\hline Female & $15(25 \%)$ \\
\hline Age (years) & 45-85 (mean: 66.1) \\
\hline \multicolumn{2}{|l|}{ Location } \\
\hline Upper & $10(16 \%)$ \\
\hline Middle & $17(28 \%)$ \\
\hline Lower & $34(56 \%)$ \\
\hline \multicolumn{2}{|l|}{ Differentiation } \\
\hline \multicolumn{2}{|l|}{ Luminal surface } \\
\hline Papillary & $4(7 \%)$ \\
\hline Tubular, well differentiated & $38(62 \%)$ \\
\hline Tubular, moderately differentiated & $17(28 \%)$ \\
\hline Tubular, poorly differentiated & $1(2 \%)$ \\
\hline Undifferentiated & $1(2 \%)$ \\
\hline \multicolumn{2}{|l|}{ Invasive front $(n=58)$} \\
\hline Papillary & $0(0 \%)$ \\
\hline Tubular, well differentiated & $16(28 \%)$ \\
\hline Tubular, moderately differentiated & $33(57 \%)$ \\
\hline Tubular, poorly differentiated & $8(14 \%)$ \\
\hline Undifferentiated & $1(2 \%)$ \\
\hline \multicolumn{2}{|l|}{ Depth of invasion ( $T$ number) } \\
\hline Tis & $3(5 \%)$ \\
\hline T1b & $1(2 \%)$ \\
\hline $\mathrm{T} 2$ & $22(36 \%)$ \\
\hline T3 & $35(57 \%)$ \\
\hline \multicolumn{2}{|l|}{ Lymphatic permeation } \\
\hline Negative & $14(23 \%)$ \\
\hline Positive & $47(77 \%)$ \\
\hline \multicolumn{2}{|l|}{ Venous invasion } \\
\hline Negative & $23(38 \%)$ \\
\hline Positive & $38(62 \%)$ \\
\hline \multicolumn{2}{|l|}{ Perineural invasion } \\
\hline Negative & $12(20 \%)$ \\
\hline Positive & $49(80 \%)$ \\
\hline \multicolumn{2}{|l|}{ Lymph node metastasis ( $N$ ) } \\
\hline Negative & $33(54 \%)$ \\
\hline Positive & $28(46 \%)$ \\
\hline \multicolumn{2}{|l|}{ TNM stage } \\
\hline 0 & $3(5 \%)$ \\
\hline I & $1(2 \%)$ \\
\hline II & $13(21 \%)$ \\
\hline III & $9(15 \%)$ \\
\hline IVA & $35(57 \%)$ \\
\hline
\end{tabular}




\section{Immunohistochemical Staining}

Immunohistochemistry was performed on paraffinembedded tissue sections using the streptavidinbiotin-complex method. Sections (3- $\mu$ m thick) of the EBDC specimens were deparaffinized in xylene and rehydrated through a graded ethanol series. After blocking of endogenous peroxidase activity by incubation in methanol containing $0.3 \% \mathrm{H}_{2} \mathrm{O}_{2}$ for $30 \mathrm{~min}$, antigen retrieval was performed by heating in $10 \mathrm{mM}$ citrate buffer ( $\mathrm{pH} 6.0$ ) at $99^{\circ} \mathrm{C}$ for $20 \mathrm{~min}$ in a microwave oven. Next, nonspecific binding was blocked by a 10-min incubation with $10 \%$ goat serum in phosphate-buffered saline, and the slides were incubated with a rabbit polyclonal antiGalNAc-T3 antibody (1:3000 dilution) at $4^{\circ} \mathrm{C}$ overnight. The antigens labeled by the primary antibody were detected with a Histofine kit (Nichirei Pharmaceutical, Tokyo, Japan) and visualized using 3,3'diaminobenzidine tetrahydrochloride as a chromogen, followed by counterstaining with hematoxylin.
Some breast carcinoma specimens were stained in parallel as positive controls, while nonimmunized rabbit IgG was used instead of the primary antibody for negative controls. All the slides were evaluated by two observers (TI and TE) who had no prior knowledge of the clinicopathological information.

\section{Statistical Analysis}

Statistical analyses were performed using StatView (Abacus Concepts Inc., Berkeley, CA, USA). Fisher's exact test and Mann-Whitney's $U$-test were used to analyze the correlations between GalNAc-T3 expression and clinicopathologic features. Kaplan-Meier methods with the log-rank test were used to calculate the overall survival rate and differences in the survival curves. The results were considered to be statistically significant when the $P$-values were less than 0.05 .
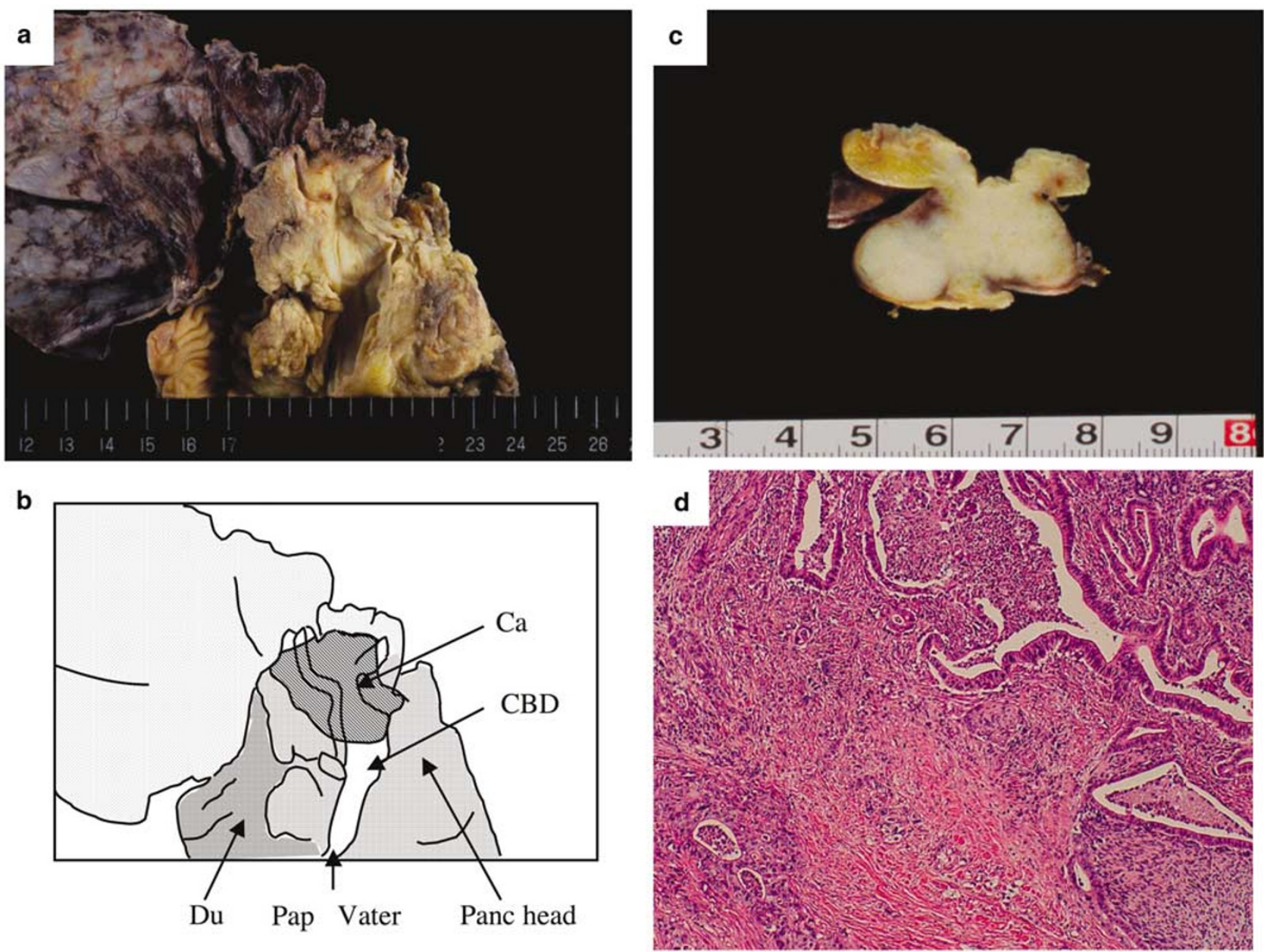

Figure 1 Representative case of a massively invasive EBDC. (a) Gross appearance and (b) mapping of the carcinoma based on histological examination of stepwise sections (Ca: carcinoma; CBD: common bile duct; Pap Vater: papilla of Vater; Panc head: pancreas head; Du: duodenum). (c) Cut surface and (d) histological features of the carcinoma. The carcinoma involves the whole thickness of the bile duct wall, and invades the surrounding fibroadipose tissue. Histologically, a well to poorly differentiated tubular adenocarcinoma is recognized. 


\section{Results}

\section{Clinicopathological Features of the EBDCs}

The study patients were 46 males and 15 females ranging in age from 45 to 85 years (mean age, 66.1 years). The clinicopathological findings based on detailed examination of whole stepwise sections of each EBDC according to the General Rules for Surgical and Pathological Studies on Cancer of the Biliary Tract and the TNM classification are summarized in Table 1. Overall, 57 (93\%) of the 61 EBDCs were massively invasive, while only three $(5 \%)$ and one $(2 \%)$ EBDCs were noninvasive and minimally invasive (invading the fibromuscular layer), respectively. In addition, two of the three noninvasive EBDCs showed a pedunculated gross appearance. Overall, the EDBCs exhibited marked lymphatic permeation (47/61 cases, $77 \%)$, venous invasion (38/61 cases, 62\%), perineural invasion (49/61 cases, 80\%) and frequent lymph node metastases (28/61 cases, 46\%). Regarding tumor differentiation, 42 of the total 61 EBDCs (69\%) were well differentiated (papillary or well differentiated tubular) at the luminal surface and 42 of the 58 minimally or massively invasive EBDCs (72\%) were poorly differentiated (moderately to poorly differentiated tubular and undifferentiated) at the invasive front. The gross and histopathological appearances of representative cases of massively invasive and noninvasive EBDCs are shown in Figures 1 and 2, respectively.

\section{Immunohistochemical Expression and Localization of GalNAc-T3 in EBDCs}

In the current study, the immunohistochemical localizations and patterns of GalNAc-T3 expression in EBDCs were investigated. The immunohistochemical patterns of GalNAc-T3 were classified into two types, namely granular and diffuse types, based on the predominant expression pattern and
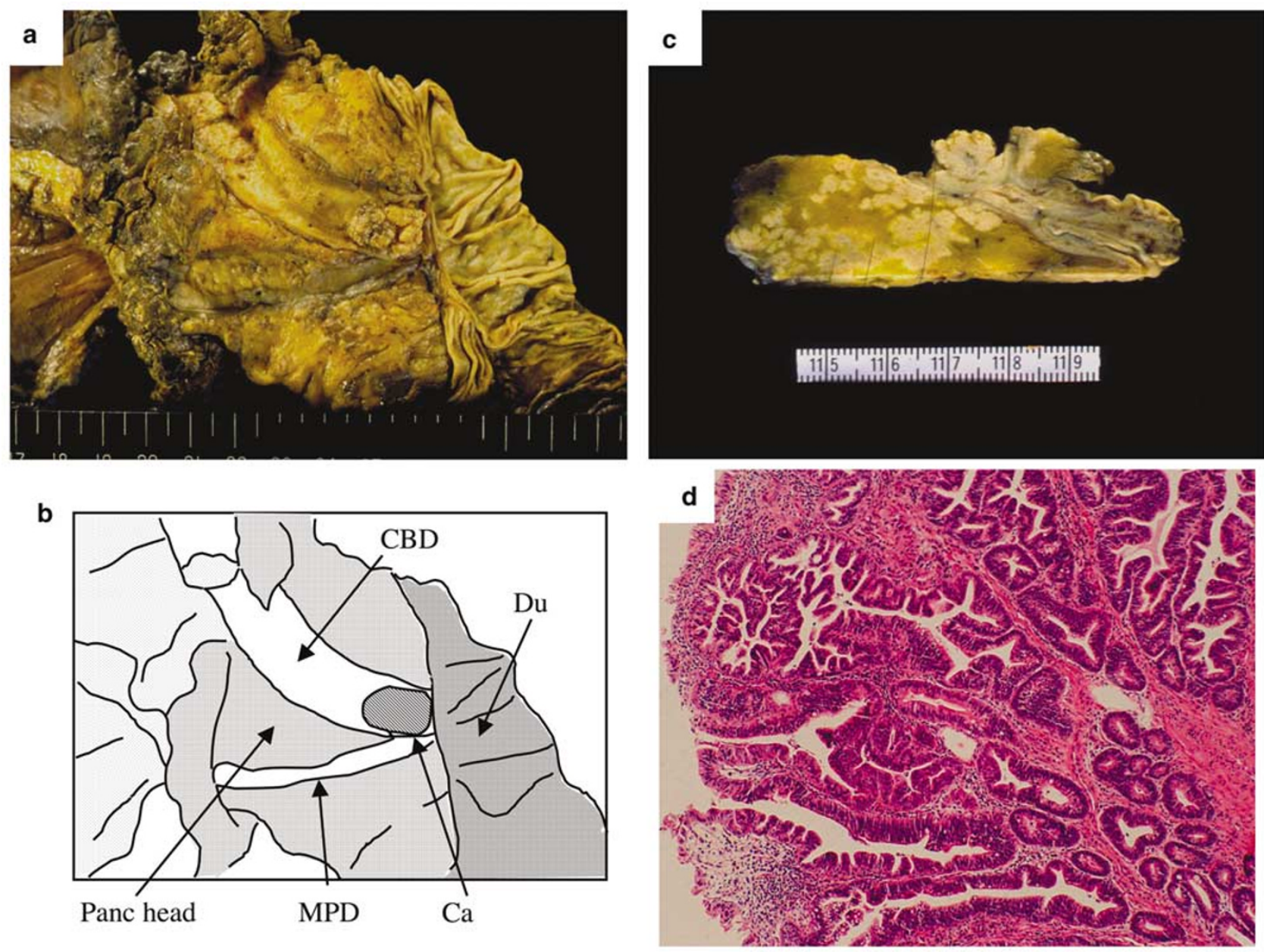

Figure 2 Representative case of a noninvasive EBDC. (a) Gross appearance and (b) mapping of the carcinoma based on histological examination of stepwise sections (Ca: carcinoma; CBD: common bile duct; MPD: main pancreatic duct; Panc head: pancreas head; Du: duodenum). (c) Cut surface and (d) histological features of the carcinoma. Grossly, the tumor is pedunculated. Histologically, a welldifferentiated papillary or tubular adenocarcinoma is recognized. The carcinoma is confined to the mucosa of the bile duct, and stromal invasion is not recognized. 
subcellular distribution. The granular type revealed GalNAc-T3 staining that was strong, granular and mostly restricted to the perinuclear areas, whereas the diffuse type revealed GalNAc-T3 staining that was diffusely but weakly positive in the cytoplasm. Figures 3 and 4 show representative examples of the granular and diffuse types of GalNAc-T3 staining pattern in EBDCs, respectively. The GalNAc-T3 expression patterns at the luminal surface and invasive front were evaluated separately if the carcinoma was invasive, and classified as the granular or diffuse type when over $50 \%$ of the cancerous epithelia in each region of the section showed the corresponding expression pattern.

All four noninvasive or minimally invasive EBDC cases showed the granular type (Figure 5). Among the 58 minimally or massively invasive EBDC cases, the GalNAc-T3 expression pattern at the luminal surface was the granular type in 38 cases $(66 \%)$ and diffuse type in 20 cases (34\%), while the expression pattern at the invasive front was the granular type in 26 cases (45\%) and diffuse type in 32 cases (55\%).
The 38 cases with the granular type at the luminal surface were divided into 26 cases of the granular type $(68 \%)$ and 12 cases of the diffuse type $(32 \%)$ at the invasive front. All 20 cases of the diffuse type at the luminal surface showed diffuse-type expression at the invasive front, and none of them revealed the granular-type expression at the invasive front (Figure 6).

\section{Relationships between the GalNAc-T3 Expression Patterns and Clinicopathological Features of EBDCs}

The correlations between the GalNAc-T3 expression patterns at the luminal surface and invasive front and the clinicopathological features in EBDCs are summarized in Table 2 .

All four noninvasive or minimally invasive carcinoma cases showed only the granular type, but the GalNAc-T3 expression pattern at the luminal surface did not affect the depth of invasion ( $\mathrm{T}$ number of the TNM classification).

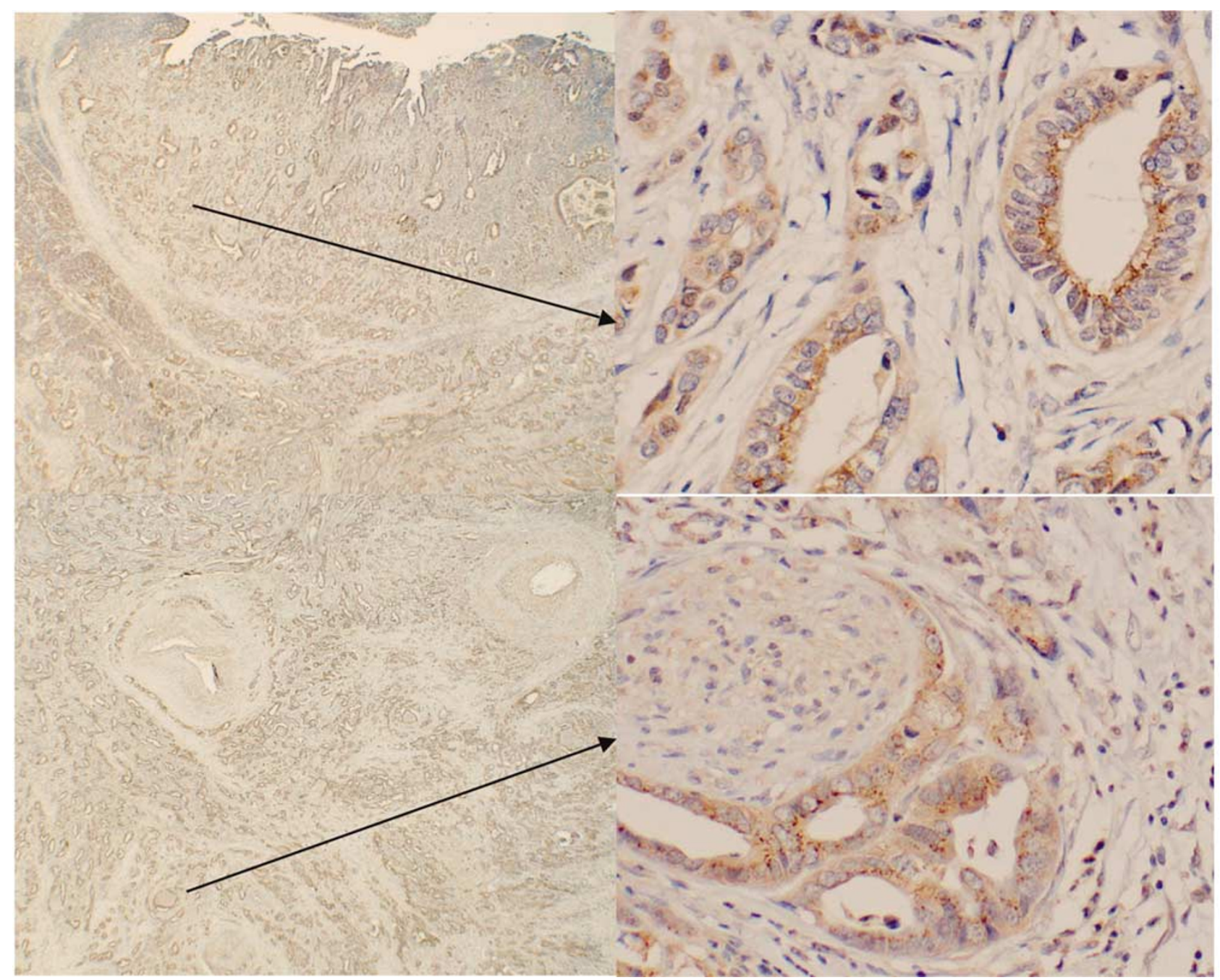

Figure 3 Representative expression pattern of GalNAc-T3 in an EBDC case. In this case, the granular-type expression at the luminal surface (top) remains the granular type at the invasive front (bottom). 


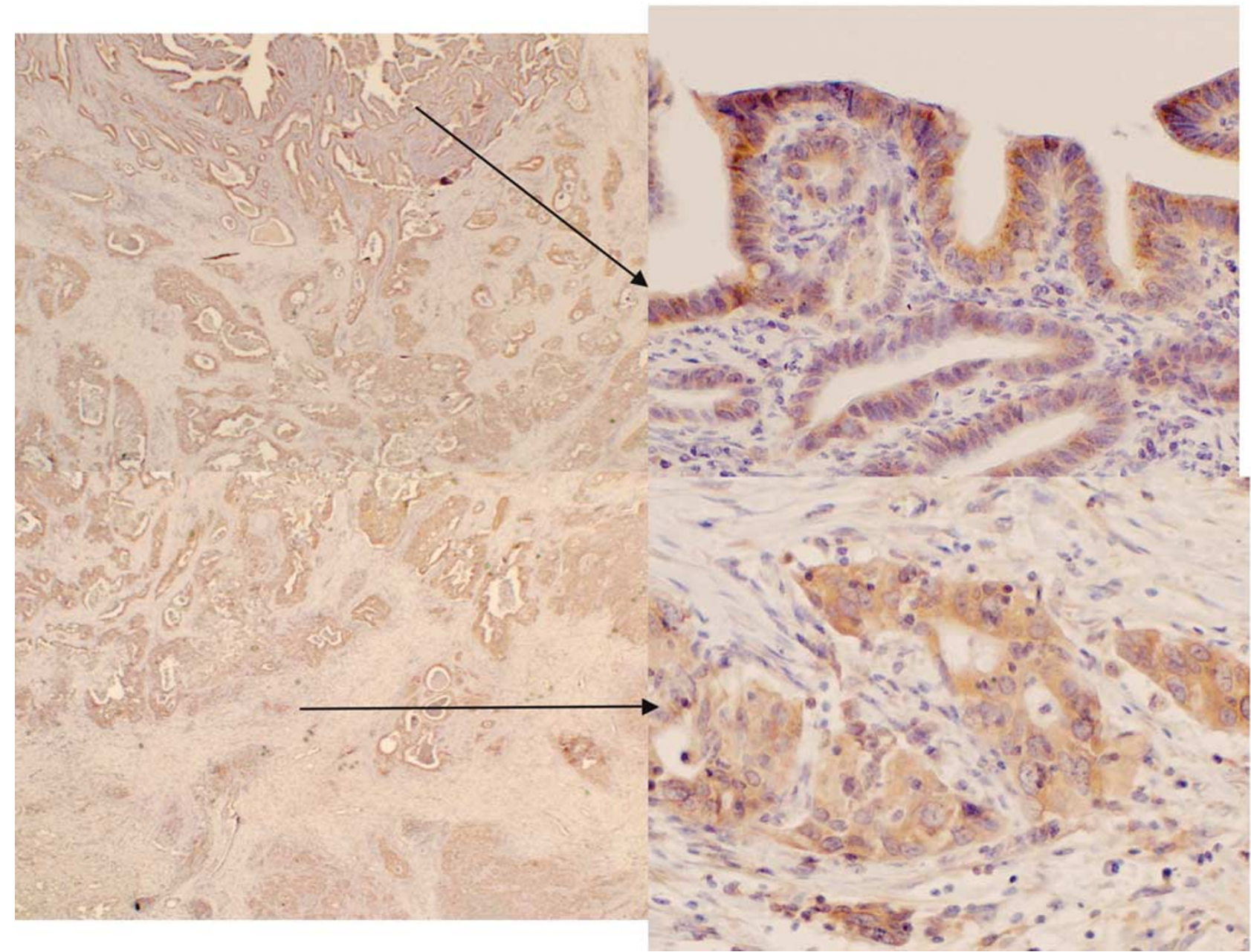

Figure 4 Representative expression pattern of GalNAc-T3 in an EBDC case. In this case, the granular-type expression at the luminal surface (top) alters to the diffuse type at the invasive front (bottom).

Cases with diffuse-type expression at the luminal surface tended to have a positive lymph node status, but the association did not reach statistical significance $(P=0.055)$. No other apparent correlations between the different GalNAc-T3 expression patterns and any of the other clinicopathological factors, such as tumor differentiation, depth of tumor invasion and angio- or perineural invasion, were detected.

The difference in GalNAc-T3 expression pattern at the invasive front did have an effect on the lymph node status of the EBDC cases. The diffuse type of GalNAc-T3 expression was associated with positive lymph node metastasis $(P=0.020)$, but no correlations with any of the other clinicopathological factors, such as tumor differentiation, depth of tumor invasion and angio- or perineural invasion, were detected.

\section{Relationships between Overall Survival in EBDCs and the Different GalNAc-T3 Expression Patterns}

The 5-year overall survival rate of all the EBDC patients was $37.7 \%$. Among the invasive EBDC patients, no statistically significant relationships between the overall survival and the different GalNAc-T3 expression patterns were detected. There was a slight tendency that the overall survival of patients with diffuse-type expression at the invasive front was better than that of patients with the granular type in contrast to the relationships between the different GalNAc-T3 expression patterns and the lymph node status (Figure 7).

\section{Discussion}

The mechanisms for the tumorigenesis, progression and biological aggressiveness of EBDCs have not yet been clarified. However, recent studies have suggested that GalNAc-T3 expression is associated with the differentiation or biological behavior of human adenocarcinomas through the initial glycosylation of mucin-type $O$-linked glycoproteins. In the present study, the relationships between the GalNAc-T3 expression patterns and the tumor characteristics of EBDCs were investigated in order to clarify the biological aggressiveness of EBDCs. 


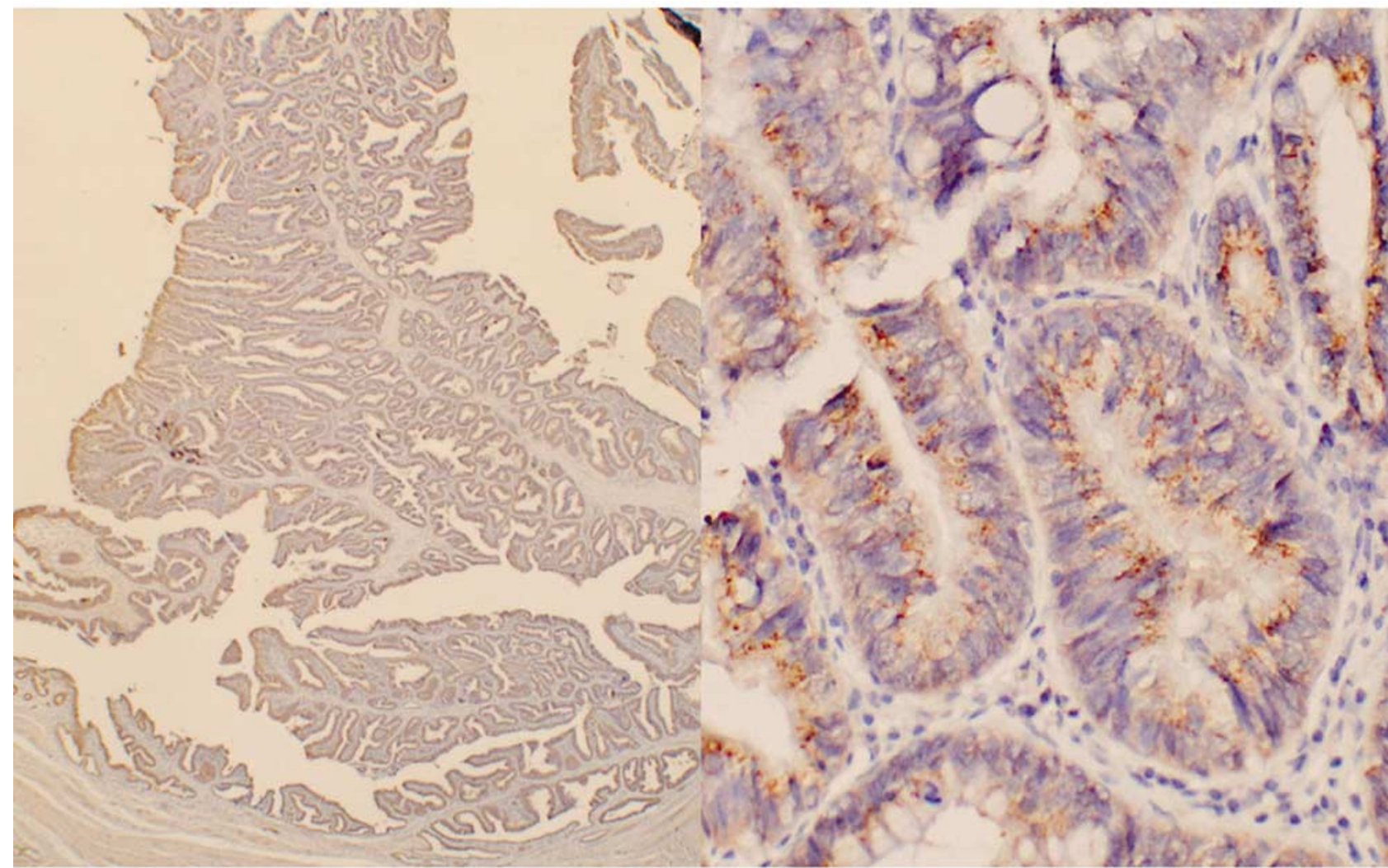

Figure 5 Granular-type expression pattern of GalNAc-T3 in a representative case of a noninvasive EBDC.

Luminal surface

Invasive front

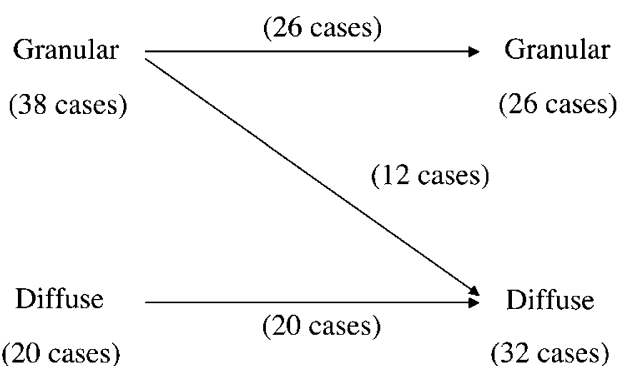

Figure 6 Alterations in the GalNAc-T3 expression patterns at the luminal surfaces and invasive fronts of the EBDCs.

The immunohistochemical expression patterns of GalNAc-T3 in our EBDC cases were classified into two types, namely the granular and diffuse types, based on the predominant expression pattern and subcellular distribution. Although most previous studies evaluating GalNAc-T3 expression by immunohistochemistry have divided the observed expression patterns into high-intensity (strong, positive) and low-intensity (weak, negative) groups, ${ }^{24-28,30,31}$ Miyahara et $a l^{29}$ divided the GalNAc-T3 expression patterns of gallbladder carcinomas into granular and diffuse types, mostly corresponding to high- intensity and low-intensity expression, respectively. We also found that the immunohistochemical staining of GalNAc-T3 in EBDCs showed granulartype and diffuse-type expression patterns, and these features of EBDCs were similar to those reported for gallbladder adenocarcinomas. ${ }^{29}$ Furthermore, our observations that all the noninvasive or minimally invasive carcinomas showed only the granular type and that the proportion of diffuse-type expression was increased at the invasive front of advanced EBDCs were also similar to the findings for gallbladder adenocarcinomas. The GalNAc-T family enzymes normally reside in the Golgi apparatus of cells, ${ }^{19}$ and alterations in the subcellular localization of GalNAc-T3 may be associated with the status of the morphological and functional differentiation of the carcinoma cells. The diffuse-type localization of GalNAc-T3 in carcinoma cells indicates a reorganization of the Golgi apparatus elements in the carcinoma cells as reported previously. ${ }^{8,19,20}$ Our data reveal further similarities of the GalNAc-T3 expression in EBDCs with that in gallbladder carcinomas, in that EBDCs alter their GalNAc-T3 expression from the granular type to the diffuse type during tumor growth. The present study also demonstrates that EBDCs are less differentiated and show diffuse-type GalNAc-T3 expression more frequently at their invasive front. However, there was no significant correlation between the 
Table 2 Relationships between GalNAc-T3 expression and clinicopathologic features of the EBDCs

\begin{tabular}{|c|c|c|c|c|c|c|}
\hline & \multicolumn{2}{|c|}{ Luminal surface } & & \multicolumn{2}{|c|}{ Invasive front } & \\
\hline & $\begin{array}{l}\text { Granular } \\
(\mathrm{n}=41)\end{array}$ & $\begin{array}{l}\text { Diffuse } \\
(\mathrm{n}=20)\end{array}$ & & $\begin{array}{c}\text { Granular } \\
(\mathrm{n}=26)\end{array}$ & $\begin{array}{l}\text { Diffuse } \\
(\mathrm{n}=32)\end{array}$ & \\
\hline \multicolumn{7}{|l|}{ Tumor differentiation } \\
\hline Papillary & 3 & 1 & NS & 0 & 0 & NS \\
\hline Tubular, well differentiated & 28 & 10 & & 9 & 7 & \\
\hline Tubular, moderately differentiated & 9 & 8 & & 15 & 18 & \\
\hline Tubular, poorly differentiated and undifferentiated & 1 & 1 & & 2 & 7 & \\
\hline \multicolumn{7}{|l|}{ Depth of invasion ( $T$ number) } \\
\hline Tis & 3 & 0 & NS & 0 & 0 & NS \\
\hline T1b & 1 & 0 & & 1 & 0 & \\
\hline $\mathrm{T} 2$ & 14 & 8 & & 10 & 12 & \\
\hline $\mathrm{T} 3$ & 23 & 12 & & 15 & 20 & \\
\hline \multicolumn{7}{|l|}{ Lymphatic permeation } \\
\hline Negative & 12 & 2 & NS & 6 & 5 & NS \\
\hline Positive & 29 & 18 & & 20 & 27 & \\
\hline \multicolumn{7}{|l|}{ Venous invasion } \\
\hline Negative & 18 & 5 & NS & 9 & 11 & NS \\
\hline Positive & 23 & 15 & & 17 & 21 & \\
\hline \multicolumn{7}{|l|}{ Perineural invasion } \\
\hline Negative & 9 & 3 & NS & 4 & 5 & NS \\
\hline Positive & 32 & 17 & & 22 & 27 & \\
\hline \multicolumn{7}{|l|}{ Lymph node metastasis } \\
\hline Negative & 26 & 7 & $P=0.055$ & 18 & 12 & $P=0.02^{*}$ \\
\hline Positive & 15 & 13 & & 8 & 20 & \\
\hline \multicolumn{7}{|l|}{ TNM stage } \\
\hline 0 & 3 & 0 & & & & \\
\hline $\mathrm{I}$ & 1 & 0 & & 1 & 0 & \\
\hline II & 10 & 3 & NS & 8 & 5 & NS \\
\hline III & 4 & 5 & & 2 & 7 & \\
\hline IVA & 23 & 12 & & 15 & 20 & \\
\hline
\end{tabular}

${ }^{*}$ Statistically significant.

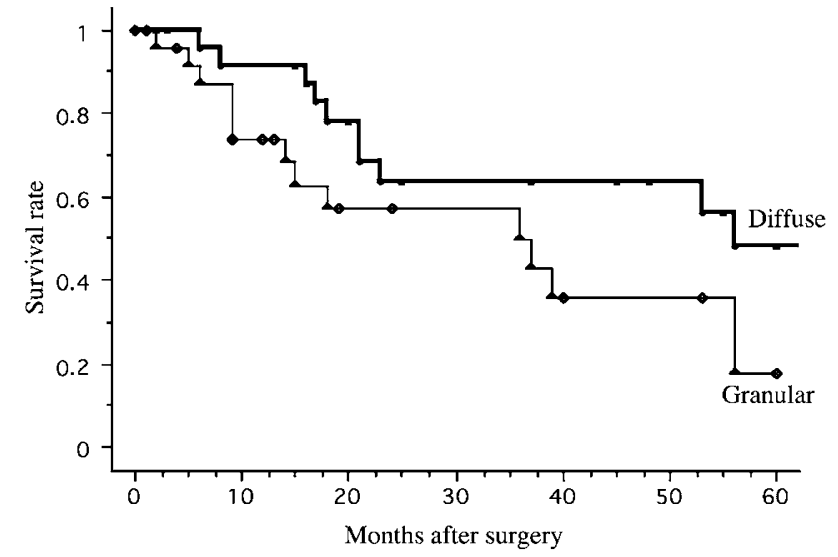

Figure 7 Relationships between the survival outcome and GalNAc-T3 expression patterns at the invasive fronts of the EBDC cases evaluated by Kaplan-Meier's survival curves.

GalNAc-T3 expression and tumor differentiation. The above facts indicate that altered subcellular localization of GalNAc-T3 cannot be explained solely by differences in the tumor differentiation of EBDCs.

In our investigation, GalNAc-T3 was weakly to moderately expressed with a granular pattern in noncancerous bile duct epithelium, compared with the strong positivity of noncarcinomatous colorectal epithelium and negativity of breast glandular tissue reported in previous studies. ${ }^{18,24}$ Colorectal carcinomas showed a lower intensity of GalNAc-T3 expression than normal colorectal epithelium, ${ }^{24}$ while breast carcinomas showed a higher intensity than normal breast glandular tissue. ${ }^{18}$ Furthermore, in the current study, the diffuse type of GalNAc-T3 expression in EBDCs was associated with positive lymph node metastasis. In contrast, differences in GalNAc-T3 expression did not have any significant effect on the overall survival of EBDC patients, although there was a slight tendency that the overall survival of patients with diffuse-type expression was better than that of patients with granulartype expression. In the adenocarcinomas of some organs, including the pancreas and gallbladder, the presence of weak expression or diffuse-type 
localization of GalNAc-T3 was correlated with tumor aggressiveness. ${ }^{24-29}$ On the other hand, early-stage gastric carcinomas with submucosal invasion tended to show positive (strong or granular) GalNAc-T3 expression, while early-stage undifferentiated gastric carcinomas showing positive GalNAc-T3 expression were associated with significantly higher frequencies of metastatic lymph node involvement. $^{30}$ In addition, GalNAc-T3-positive esophageal squamous cell carcinomas exhibited more aggressive behavior. ${ }^{31}$ It has been reported that some O-linked carbohydrate or mucin antigens affect the metastatic processes of malignant tumors, such as escape from the primary tumor, vessel invasion and migration. ${ }^{8}$ The initial glycosylation of $O$-linked carbohydrate antigens is regulated by differential expression of GalNAc-T subtypes. Therefore, differential GalNAc-T expression may also play a key role in the metastatic processes of malignant tumors. However, since the functions of the GalNAc-T family, as well as their associations and mechanisms in the metastatic processes of malignant tumors, have not yet been completely elucidated, it is currently difficult to explain how the various correlations between different GalNAc-T expression patterns and the biological behavior of malignant tumors in various organs occur. Differences in the results from EBDCs and other types of carcinomas are partly, but not completely, explained by the dissimilar characters of the carcinomas themselves. Further investigations into the GalNAc-T3 expression patterns in the carcinomas of different organs as well as the functions of this enzyme are necessary.

Regarding the GalNAc-T3 expression in EBDCs from the clinical aspect, we consider that, if a preoperative biopsy is taken, evaluation of the GalNAc-T3 expression in sections of the biopsy is useful for predicting the lymph node status preoperatively. Our present results have demonstrated that cases with diffuse-type expression at the luminal surface tend to have a positive lymph node status, while those with diffuse-type expression at the invasive front are significantly correlated with positive lymph node metastasis. All the cases with diffuse-type expression at the luminal surface also showed diffuse-type expression at the invasive front. Therefore, when a preoperative EBDC biopsy specimen shows the diffuse type of GalNAc-T3 expression pattern, the patient is predicted to carry lymph node metastasis with a higher probability than patients with granular-type GalNAc-T3 expression, and it is necessary to plan extensive lymph node dissection during the surgical treatment. Therefore, preoperative evaluation of GalNAc-T3 expression in EBDCs is useful for predicting the lymph node status and planning surgical procedures.

In conclusion, expression of GalNac-T3 in EBDCs was evaluated and compared with the clinicopathologic features in the present study. The immuno- histochemical expression patterns of GalNAc-T3 in EBDCs were classified into two types, namely granular and diffuse types. EBDCs altered their GalNAc-T3 expression during tumor growth, and this difference in the GalNAc-T3 expression pattern was considered to be associated with the mode of tumor progression, such as lymph node metastasis. Clinically, a preoperative evaluation of GalNAc-T3 expression is considered to be useful for planning operative procedures.

\section{References}

1 Michaud DS. The epidemiology of pancreatic, gallbladder, and other biliary tract cancers. Gastrointest Endosc 2002;56:S195-S200.

2 Henson DE, Albores-Saavedra J, Corle D. Carcinoma of the extrahepatic bile ducts. Histologic types, stage of disease, grade, and survival rates. Cancer 1992;70: 1498-1501.

3 Yamaguchi K, Chijiiwa K, Saiki S, et al. Carcinoma of the extrahepatic bile duct: mode of spread and its prognostic implications. Hepatogastroenterology 1997; 44:1256-1261.

4 Suda K, Miyano T, Konuma I, et al. An abnormal pancreatico-choledocho-ductal junction in cases of biliary tract carcinoma. Cancer 1983;52:2086-2088.

5 Nagata E, Sakai K, Kinoshita H, et al. Choledochal cyst: complications of anomalous connection between the choledochus and pancreatic duct and carcinoma of the biliary tract. World J Surg 1986;10:102-110.

6 Matsubara T, Sakurai Y, Sasayama Y, et al. K-ras point mutations in cancerous and noncancerous biliary epithelium in patients with pancreaticobiliary maljunction. Cancer 1996;77:1752-1757.

7 Matsubara T, Funabiki T, Jinno O, et al. p53 gene mutations and overexpression of p53 product in cancerous and noncancerous biliary epithelium in patients with pancreaticobiliary maljunction. J Hepatobiliary Pancreat Surg 1999;6:286-293.

8 Brockhausen I. Pathways of $O$-glycan biosynthesis in cancer cells. Biochim Biophys Acta 1999;1473:67-95.

9 Homa FL, Hollander T, Lehman DJ, et al. Isolation and expression of a cDNA clone encoding a bovine UDPGalNAc:polypeptide $N$-acetylgalactosaminyltransferase. J Biol Chem 1993;268:12609-12616.

10 Hagen FK, Van Wuyckhuyse B, Tabak LA. Purification, cloning, and expression of a bovine UDP-GalNAc: polypeptide $N$-acetyl-galactosaminyltransferase. J Biol Chem 1993;268:18960-18965.

11 Wandall HH, Hassan H, Mirgorodskaya E, et al. Substrate specificities of three members of the human UDP- $N$-acetyl-alpha-D-galactosamine:polypeptide $\mathrm{N}$ acetylgalactosaminyltransferase family, GalNAc-T1, -T2, and -T3. J Biol Chem 1997;272:23503-23514.

12 White T, Bennett EP, Takio K, et al. Purification and cDNA cloning of a human UDP- $N$-acetyl-alpha-Dgalactosamine:polypeptide $\quad N$-acetylgalactosaminyltransferase. J Biol Chem 1995;270:24156-24165.

13 Bennett EP, Hassan H, Clausen H. cDNA cloning and expression of a novel human UDP- $N$-acetyl-alpha-Dgalactosamine. Polypeptide $N$-acetylgalactosaminyltransferase, GalNAc-T3. J Biol Chem 1996;271: 17006-17012. 
14 Ten Hagen KG, Hagen FK, Balys MM, et al. Cloning and expression of a novel, tissue specifically expressed member of the UDP-GalNAc:polypeptide $N$-acetylgalactosaminyltransferase family. J Biol Chem 1998;273: 27749-27754.

15 Bennett EP, Hassan H, Mandel U, et al. Cloning and characterization of a close homologue of human UDP$N$-acetyl-alpha-D-galactosamine:polypeptide $N$-acetylgalactosaminyltransferase-T3, designated GalNAc-T6. Evidence for genetic but not functional redundancy. J Biol Chem 1999;274:25362-25370.

16 Cheng L, Tachibana K, Iwasaki H, et al. Characterization of a novel human UDP-GalNAc transferase, pp-GalNAc-T15. FEBS Lett 2004;566:17-24.

17 Sutherlin ME, Nishimori I, Caffrey T, et al. Expression of three UDP- $N$-acetyl-alpha-D-galactosamine:polypeptide GalNAc $N$-acetylgalactosaminyltransferases in adenocarcinoma cell lines. Cancer Res 1997;57: 4744-4748.

18 Nomoto M, Izumi H, Ise T, et al. Structural basis for the regulation of UDP- $\mathrm{N}$-acetyl-alpha-D-galactosamine:polypeptide $N$-acetylgalactosaminyl transferase-3 gene expression in adenocarcinoma cells. Cancer Res 1999;59:6214-6222.

19 Roettger S, White J, Wandall HH, et al. Localization of three human polypeptide GalNAc-transferases in HeLa cells suggests initiation of $O$-linked glycosylation throughout the Golgi apparatus. J Cell Sci 1998;111: 45-60.

20 Taatjes DJ, Roth J, Weinstein J, et al. Codistribution of galactosyl- and sialyltransferase: reorganization of trans Golgi apparatus elements in hepatocytes in intact liver and cell culture. Eur J Cell Biol 1987;44: 187-194.

21 Japanese Society of Biliary Surgery. General Rules for Surgical and Pathological Studies on Cancer of the Biliary Tract, 5th edn. Kanehara Shuppan: Tokyo, 2003.

22 Albores-Saavedra J, Henson DE, Klimstra DS. Tumors of the gallbladder, extrahepatic bile ducts and ampulla of Vater. In: Rosai J (ed). Atlas of Tumor Pathology, 3rd series, Armed Forces Institute of Pathology: Washington, 2000.

23 Albores-Saavedra J, Scoazec JC, Wittekind C, et al. Carcinoma of the gallbladder and extrahepatic bile duct. In: Hamilton SR, Aaltonen LA (eds). World Health Organization Classification of Tumours. Tumours of the Digestive System. IARC Press: Lyon, 2000, pp 206-214.

24 Shibao K, Izumi H, Nakayama Y, et al. Expression of UDP-N-acetyl-alpha-D-galactosamine-polypeptide galNAc N-acetylgalactosaminyl transferase-3 in relation to differentiation and prognosis in patients with colorectal carcinoma. Cancer 2002;94: 1939-1946.

25 Dosaka-Akita H, Kinoshita I, Yamazaki K, et al. Nacetylgalactosaminyl transferase-3 is a potential new marker for non-small cell lung cancers. Br J Cancer 2002;87:751-755.

26 Onitsuka K, Shibao K, Nakayama Y, et al. Prognostic significance of UDP-N-acetyl-alpha-D-galactosamine: polypeptide $\mathrm{N}$-acetylgalactosaminyltransferase-3 (GalNAc-T3) expression in patients with gastric carcinoma. Cancer Sci 2003;94:32-36.

27 Yamamoto S, Nakamori S, Tsujie M, et al. Expression of uridine diphosphate $N$-acetyl-alpha-D-galactosamine:polypeptide $N$-acetylgalactosaminyl transferase 3 in adenocarcinoma of the pancreas. Pathobiology 2004; 71:12-18.

$28 \mathrm{Gu} \mathrm{C}$, Oyama T, Osaki T, et al. Low expression of polypeptide GalNAc $N$-acetylgalactosaminyl transferase-3 in lung adenocarcinoma: impact on poor prognosis and early recurrence. Br J Cancer 2004;90: 436-442.

29 Miyahara N, Shoda J, Kawamoto T, et al. Expression of UDP- $N$-acetyl-alpha-D-galactosamine-polypeptide $\mathrm{N}$-acetylgalactosaminyltransferase isozyme 3 in the subserosal layer correlates with postsurgical survival of pathological tumor stage 2 carcinoma of the gallbladder. Clin Cancer Res 2004;10:2090-2099.

30 Ishikawa M, Kitayama J, Kohno K, et al. The expression pattern of UDP- $N$-acetyl-alpha-D-galactosamine: polypeptide $N$-acetylgalactosaminyl transferase-3 in early gastric carcinoma. J Surg Oncol 2004;86: 28-33.

31 Ishikawa M, Kitayama J, Nariko H, et al. The expression pattern of UDP- $N$-acetyl-alpha-D-galactosaminepolypeptide $N$-acetyl-galactosaminyl transferase- 3 in squamous cell carcinoma of the esophagus. Pathobiology 2005;72:139-145. 